\title{
The Phenomenon of the "First Stress Peak" at the Yielding in the Dynamic Compression Tests of FCC and BCC Microalloyed Structures
}

\author{
Remigiusz Błoniarz ${ }^{*}$, Janusz Majta ${ }^{1}$, Carl P. Trujillo ${ }^{2}$, Ellen K. Cerreta ${ }^{2}$ and Krzysztof \\ Muszka $^{1}$ \\ ${ }^{1}$ AGH University of Science and Technology, Department of Metals Engineering and Industrial \\ Computer Science, Mickiewicza 30, 30-059 Krakow, Poland \\ ${ }^{2}$ MST, Los Alamos National Laboratory, Los Alamos MS755, NM, USA
}

\begin{abstract}
In the presented work two grades of steel i.e. microalloyed ferrite (M_F) and microalloyed austenite (M_A) where subjected to the grain refinement processes using MaxStrain system and thermomechanical rolling. The wide range of grain size, starting from $200 \mu \mathrm{m}$ down to submicrometer level was produced in this way. The specimens of both steels were subjected to the dynamic compression tests using the Split Hopkinson Pressure Bar (SHPB) apparatus and applying the strain rates in the range between $\dot{\varepsilon}=3750 \mathrm{~s}^{-1}$ and $\dot{\varepsilon}=6000 \mathrm{~s}^{-1}$. In addition, different temperatures were used in the tests, i.e. $200{ }^{\circ} \mathrm{C}$ and $400{ }^{\circ} \mathrm{C}$. The first peak of stress which is observed during elastic-plastic transition during the dynamic compression tests can be treated as a characteristic feature of the tested material. The results obtained in the present investigations showed a significant dependence of the "first stress peak" in the dynamic compression curve on the degree of the microstructure refinement for the samples of M_F and almost complete absence of this dependence for M_A.
\end{abstract}

The phenomenon of the first peak of stress which is observed during elastic-plastic transition during the dynamic compression tests can be treated as a characteristic feature of the tested material. So far, the reasons for this phenomenon have not been sufficiently explained. A first look at the stress-strain curve suggests some similarity to the upper yield stress and Lüders strain, but the absence of an apparent strain in the yield stress region points to a different cause. Likewise, the unblocking effect of the so-called Cottrell atmospheres also does not seem to be a good explanation.

The first attempts to explain this typical effect, observed in both coarse and ultra-fine grained (UFG) structures, have been proposed by Carrington et al. in 1948 [1]. It was proposed to link the appearance of the first peak of stress on the dynamic compression curve with individual local block movement in particular grains. It has been observed that the boundaries between these rigid blocks further becomes twins or deformation bands. However, this hypothesis does not explain why one group of the metallic materials exhibits this characteristic increase in the initial plastic stress of the material and another does not.

\footnotetext{
* Corresponding author: bloniarz@agh.edu.pl
} 
The main goal of the present study was to provide arguments for explaining the phenomenon of the first peak on the stress-strain curve during dynamic compression. It should be expected that this will allow to define conditions for stable plastic flow during deformation of such popular construction materials as modern microalloyed steels, additionally characterized by an ultrafine-grained microstructure. Achieving this goal will not only improve the stability of metal forming processes carried out at very high deformation rates, but will also contribute to improving the safety of steel structures subjected to dynamic loading conditions e.g. in the automotive industry, mining, etc.

\section{Experimental}

The research materials used in this study were samples made of two microalloyed steels in the form of hot-rolled billets, the chemical composition of which are: $\mathrm{M} \_\mathrm{A}-0.05 \% \mathrm{C}$, $1.64 \% \mathrm{Mn}, 0.3 \% \mathrm{Si}, 0.07 \% \mathrm{Ti}, 0.037 \% \mathrm{Nb}, 30.8 \% \mathrm{Ni}, 0.004 \% \mathrm{~N}$; and for M_F: $0.07 \% \mathrm{C}$, $1.36 \% \mathrm{Mn}, 0.27 \% \mathrm{Si}, 0.03 \% \mathrm{Ti}, 0.067 \% \mathrm{Nb}, 0.009 \% \mathrm{~N}$. Both tested materials i.e. microalloyed austenite (M_A) and microalloyed ferrite (M_F), were subjected to a severe plastic deformation (SPD) process using the MaxStrain [2] system, integrated with a thermomechanical simulator Gleeble. The samples (initial dimensions: 10x10x27 mm) were deformed at room temperature with the total cumulative deformation $\varepsilon=10$ or $\varepsilon=20$, and then annealed by resistance heating immediately after deformation at the temperature of 500 ${ }^{\circ} \mathrm{C}$ for 1200 s. In this way, by creating conditions for the occurrence of continuous recrystallization (in situ), a strong microstructure refinement of both materials was achieved. After this processing some material was set aside for experiments ans some was reserved for further processing; specifically it was subjected to hot thermomechanical rolling in order to produce wire rod with a diameter of $6.5 \mathrm{~mm}$.

All materials were subjected to microstructural analysis in order to assess the grain size and crystallographic orientation using optical microscopy, the SEM-EBSD (Scanning Electron Microscopy - Electron Back Scattering Diffraction) method and TEM (Transmission Electron Microscopy) observation. EBSD analysis was performed with the following parameters: step $=150 \mathrm{~nm}$, min. pixels accounting for one grain $=8$, boundaries misorientation range assumed $2^{\circ} \div 15^{\circ}$ for the low angle grain boundaries, $>15^{\circ}$ for high angle grain boundaries. Then samples for mechanical testing were taken from the central part of the materials after deformation in the MaxStrain system. Specimens subjected to the SHPB tests (Split Hopkinson Pressure Bar) in form of $\phi 2,5 \times 2,5 \mathrm{~mm}$ cylinders were extracted (after previous examination for discontinuity and failure presence) from central part of the MaxStrain samples. SHPB apparatus consisted of the incident and transmitted 9,46 mm diameter bars made of maraging 350 steel. 152,4 mm length striker was launched with breech pressure $24,13 \mathrm{kPa}(3,5 \mathrm{psi})$. Strain rates achieved during tests were in the range of $3750 \mathrm{~s}^{-1}$ to $6000 \mathrm{~s}^{-1}$.

\section{Results and discussion}

The average grain size of the tested materials, estimated on the basis of optical microscopy observations, after hot thermomechanical rolling, was $15 \mu \mathrm{m}$ for both the M_A and M_F samples. The microstructure of samples subjected to the SPD process, i.e. deformed in the MaxStrain system, was analyzed in more detail, using the EBSD method. Euler maps with the high-angle (black) and low-angle (red) boundaries marked as well as the histograms of the boundary and grain size misorientation angle are presented in Fig.1. The results obtained from microstructural observations show clear differences between the state of the microstructure after MaxStrain deformation for both steels, although the average grain size 


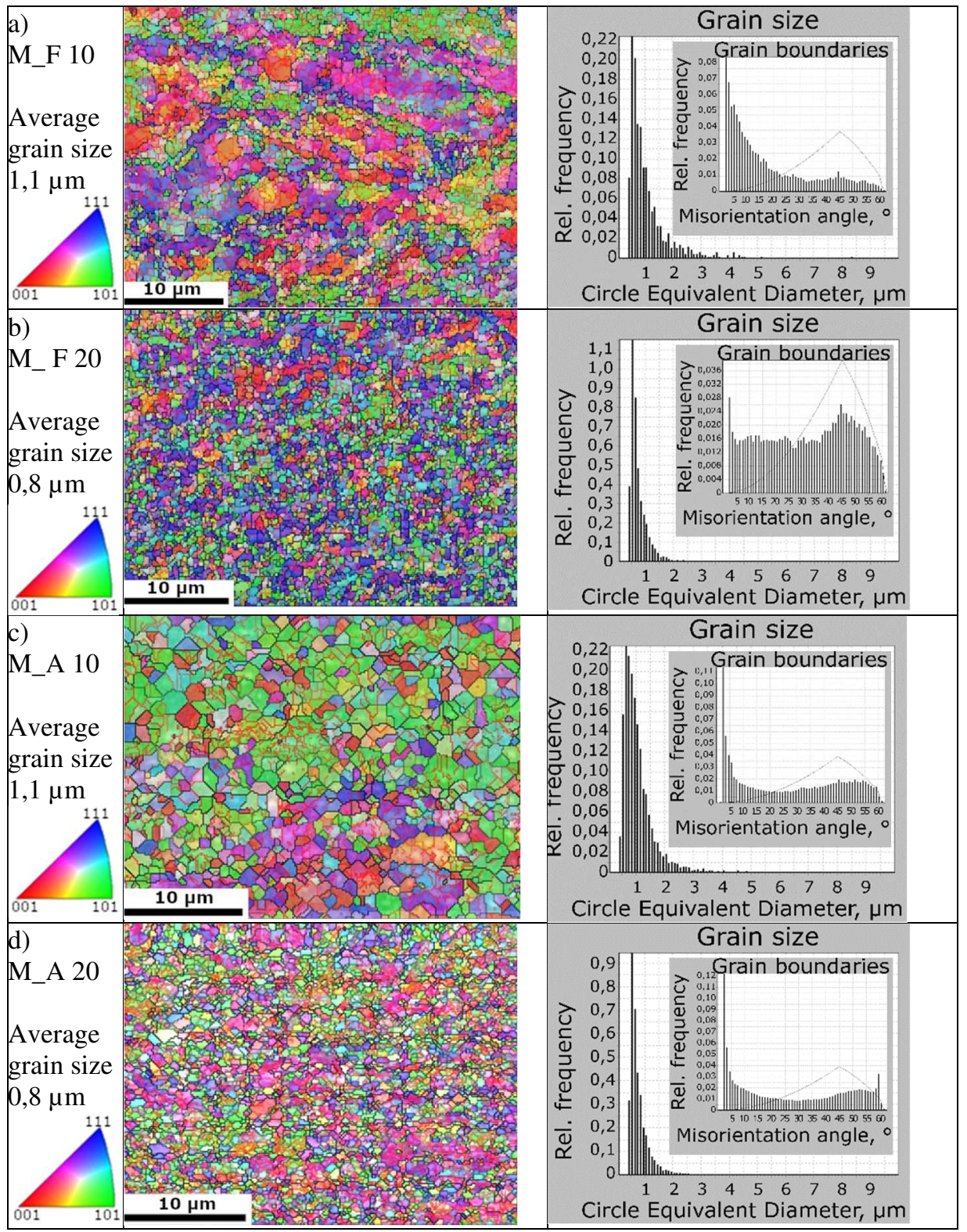

Fig. 1. Results of microstructural analysis of the samples produced by the MaxStrain system. Second column - IPF maps with marked high-angle (black) and low-angle (red) boundaries. Third column histograms of boundaries misrientation angle and grain size distribution.

is similar. In the case of the M_F samples, a strong dislocation substructure first formed (Fig. 1a), which then transformed into microstructures with large-angle grain boundaries as the strain increased (Fig. 1b). As a result, in a sample subjected to a total deformation $\varepsilon=20$, the microstructure consists of grains that are almost devoid of free dislocations and dislocation substructure and a fully isotropic distribution of grain orientation. In the case of samples of M_A steel, the cell dislocation substructure and the process of the misorientation angle growth in the walls of the dislocation cells proceed continuously during deformation, hence 
the distribution and ratio of low and high angle grain boundaries is similar for the cumulative deformation 10 and 20.

In the next stage of the study, samples with a cylindrical shape cut from materials processed in the MaxStrain system, were tested in the compression under dynamic loading conditions in the SHPB apparatus. The use of similar deformation procedures in the MaxStrain system for both tested steels made it possible to compare the mechanical response under dynamic loading conditions of microalloyed austenite and microalloyed ferrite.

a)

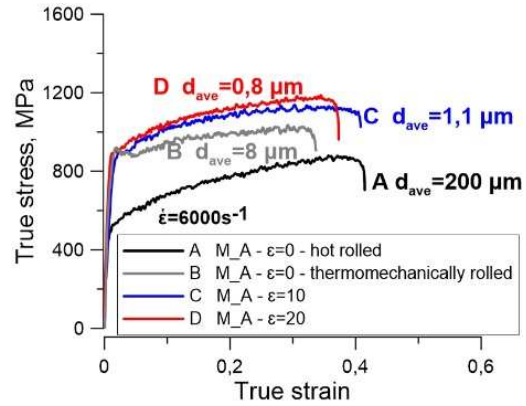

b)

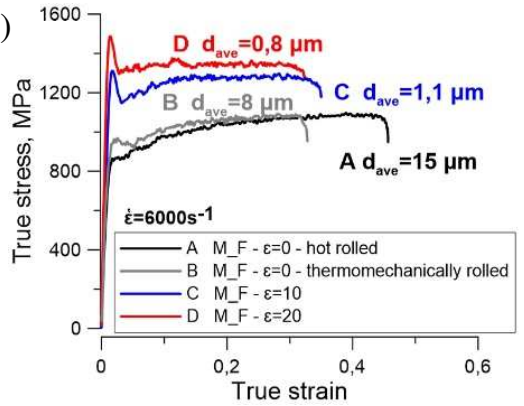

Fig.2. Comparison of the stress-strain curves obtained in the dynamic compression tests of microalloyed austenite (M_A) -a) and microalloyed ferrite (M_F) -b) as a function of the microstructure state and refinement. The symbol $\varepsilon$ represents the total strain applied during the MaxStrain processing.

The results obtained in the present investigations showed a significant dependence of the "first stress peak" in the compression curve on the degree of the microstructure refinement for the samples of M_F and almost complete absence of this dependence for M_A. From the analysis of the stress-strain curves obtained from the compression tests presented in Fig. 2, it can be seen that in the case of M_A samples, the microstructure refinement, affects the rate of work hardening and yield stress of the materials. In the case of M_F samples, the refined microstructure causes a significant increase in the yield point, without significantly affecting the rate of work hardening. Similar observations were made in previous studies of UFG microalloyed steels subjected to dynamic deformation processes [3, 4].

The conducted research confirmed the earlier observations that in the case of M_F, with the bcc lattice structure, during the transition from elastic to plastic state under the conditions of dynamic deformation, a certain peculiarity in the form of an intense first stress peak was observed. The results of the present research have shown a clear relationship between the degree of microstructure refinement and the intensity of such a peak. The M_F samples with coarse-grained microstructure $(\mathrm{d}=200 \mu \mathrm{m})$ after hot rolling show a steadily increasing hardening profile across the entire strain range. A sample cut from thermomechanically rolled wire rod shows a slight increase in stress in the area of initial plastic flow. The ultra-finegrained material, after deformation in the MaxStrain system, shows a clear and sharp peak. On the other hand, in the case of austenitic steel, only a small representation of this phenomenon is observed in the samples cut from thermomechanically rolled wire rod, but this effect is definitely smaller than in the case of the M_F sample. The presence of the first stress peak on the flow curve in the case of the M_F sample, with the total accumulated strain $\varepsilon=10$, excludes the hypothesis that the cause of this peak is the presence of a dislocation substructure because, as shown in Fig. 1 the microstructure is mostly free of dislocations. However, another material characteristic that may affect the presence of the peaks is the presence of Cottrell's atmospheres [5]. However, the dynamic loading conditions of the test seem to eliminate this explanation. Comparison of the results of SHPB compression tests on the materials in this study with low-carbon steel (LC), with the basic chemical composition of $0.03 \% \mathrm{C} ; 0.37 \% \mathrm{Mn} ; 0.14 \% \mathrm{Cu}$, was performed in order to evaluate the effect of the presence of dispersive precipitates of $\mathrm{Nb}$ carbonitrides on the occurrence of the first stress 
peak. The comparison of the curves of both steels is presented in Fig. 3 a. The presented results show that the presence of dispersion precipitates in the tested microalloyed steel most likely affects the rate of strain hardening but does not significantly affect the peak value at the yield point.

The analysis of the grain size effect suggests that the "first stress peak" is an effect mainly dependent on the microstructure, but the influence of the testing conditions was not completely understood. For this reason, other tests were carried out covering various parameters that incidentally mimic aspects of the deformation process, such as temperature and strain rate. In this way aspects of the MaxStrain deformation process were probed, The M_F 20 (subjected to the total strain of 20 on the MaxStrain system) samples were subjected to compression tests at elevated temperatures. The obtained results are shown in Fig. $3 \mathrm{~b}$ ). It has been observed that the magnitude of the peak stress decreases and becomes less sharp with increasing test temperature. While, at the test temperature of $200{ }^{\circ} \mathrm{C}$ the peak was still distinct, at $400{ }^{\circ} \mathrm{C}$, the peak is not observed. The occurrence of the first stress peak phenomenon is therefore a temperature sensitive phenomenon.

a)

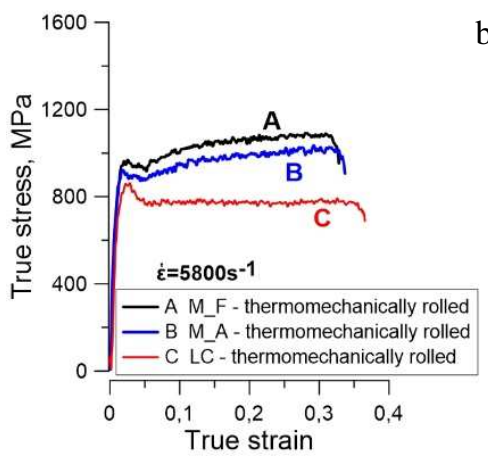

b)

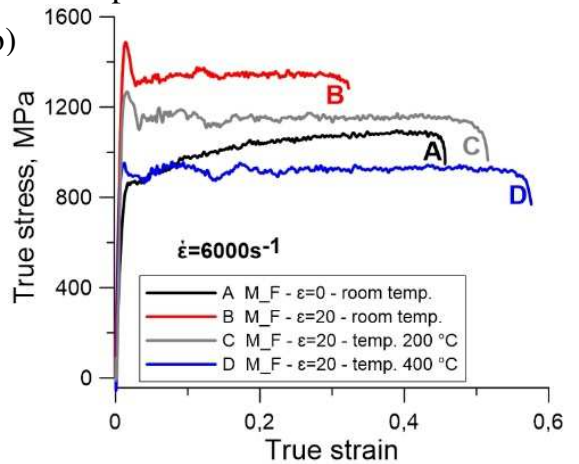

Fig. 3. Comparison of the stress-strain curves obtained in the dynamic compression tests of microalloyed austenite (M_A), microalloyed ferrite (M_F) and low carbon steel (LC) in form of wire rods-a). Stress-strain curves of M_F produced at strain rate of $6000 \mathrm{~s}^{-1}$, as function of the test temperatures of samples after MaxStrain processing.

Fig. 4 shows the initial fragments of the stress-strain curves for the M_F steel subjected to the MaxStrain procedure. The peak stress values and the corresponding strains are presented in Table 1. Especially for sample M_F 20, where the microstructure is free from the substructure, the influence of strain rate on the peak stress intensity is visible. The part of the curve showing the increase in strain hardening is quite similar, but the peak size is slightly larger for the samples tested at the higher strain rate. The figure shows the TEM results of the tested samples obtained after MaxStrain processing.

The observed influence of the deformation temperature and the sensitivity to the deformation rate suggest that in the strengthening process, an important role is played by the thermal component of the yield stress, which is typical for bcc metals. From the point of view of the microstructure, it is related to overcoming short distance obstacles.

The presented results suggest that the decrease in the grain size associated with the fragmentation of the microstructure plays a key role in shaping the peak value of the first stress. Grain boundaries are generally long-range barriers and overcoming them is associated with an increase in the athermal component of the yield stress. As a result of the strong fragmentation of the microstructure, the distances between the grain boundaries are so small that they become obstacles in close range. This means that the refinement of the microstructure increases the value of the peak stress, as does an increase in the strain rate or a decrease in the strain temperature. Therefore, if we observe an increase in peak stress and it is the effect of the thermal component of the yield stress, then this effect is related to the 


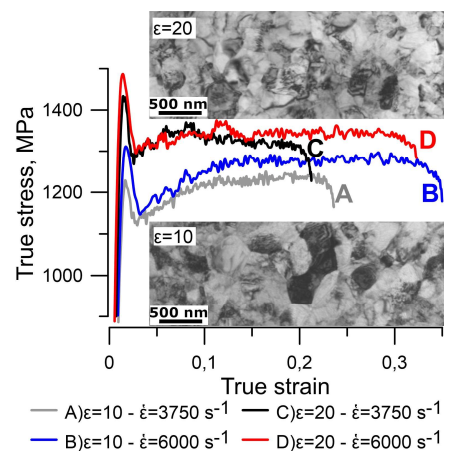

Table.1. Values for stress and strain in the highest point of the peak, and at the end of the peak.

\begin{tabular}{|c|c|c|c|c|}
\hline & $\begin{array}{l}\text { M_F; } \\
\varepsilon=10 ; \\
\dot{\varepsilon}=3750\end{array}$ & $\begin{array}{l}\text { M_F; } \\
\varepsilon=10 ; \\
\dot{\varepsilon}=6000\end{array}$ & $\begin{array}{l}\text { M_F; } \\
\varepsilon=20 ; \\
\dot{\varepsilon}=3750\end{array}$ & $\begin{array}{l}\text { M_F; } \\
\varepsilon=20 ; \\
\dot{\varepsilon}=6000\end{array}$ \\
\hline$\sigma$ max peak & $1229 \mathrm{MPa}$ & $1312 \mathrm{MPa}$ & $1433 \mathrm{MPa}$ & $1487 \mathrm{MPa}$ \\
\hline$\sigma_{\text {end peak }}$ & $1138 \mathrm{MPa}$ & $1147 \mathrm{MPa}$ & $1267 \mathrm{MPa}$ & $1295 \mathrm{MPa}$ \\
\hline$\Delta \sigma$ & $91 \mathrm{MPa}$ & $165 \mathrm{MPa}$ & $165 \mathrm{MPa}$ & $192 \mathrm{MPa}$ \\
\hline$\varepsilon$ max peak & 0,017 & 0,017 & 0,014 & 0,014 \\
\hline$\varepsilon$ end peak & 0,024 & 0,032 & 0,026 & 0,028 \\
\hline$\Delta \varepsilon$ & 0,007 & 0,015 & 0,012 & 0,014 \\
\hline
\end{tabular}

Fig. 4. The initial fragments of stress-strain curves. M_F 20 specimens SHPB test with various strain rates. Additionally TEM microstructures from MaxStrain with accumulated strain $\varepsilon=10$ and $\varepsilon=20$.

short-range barriers in the vicinity of the grain boundaries. Other long-range barriers, such as precipitates, do not work in this way, so we expect that defects and structure near the boundaries may be impacting the response,

\section{Conclusions}

The results obtained in the present investigations showed a significant dependence of the "first stress peak" in the compression curve on the degree of the microstructure refinement for the samples of M_F and almost complete absence of this dependence for M_A. The conducted analysis of the test results allows for the conclusion that the occurrence of the first peak on the dynamic compression curve at the moment of transition from elastic to plastic state is a direct effect of the increase in the density of geometrically necessary dislocations, especially in the bcc structures. This conclusion seems to be also supported by the obtained results of microstructural analysis using the EBSD method and the observations of dislocation substructures for the tested materials and deformation conditions. Results of the tests carried out at elevated temperatures suggest, that the peak stress at the beginning of yielding of the M_F specimen is a temperature sensitive phenomenon, likely associated with relaxation of the dislocations stored within the structures.

\section{References}

[1] W. E. Carrington, L. V. Marie and D. S. Gayler, The use of flat-ended projectiles for determining dynamic yield stress. III. "Changes in microstructure caused by deformation under impact at high-striking velocities" Proc. R. Soc. London, A. Math. Phys. Sci., vol. 194. pp. 323-31, (1948)

[2] W. C. Chen, D. Ferguson, H. . Ferguson, R. S. Mishra, and Z. Jin, Development of Ultrafine Grained Materials Using The MAXStrain ${ }^{\circledR}$ Technology, Mater. Sci. Forum, vol. 357-359, pp. 425-430 (2001)

[3] R. Bloniarz, J. Majta, C.P. Trujillo and E. K. Cerreta, The mechanical response of UFG and nanostructured microalloyed steels subjected to dynamic loading conditions, EPJ Web of Conf. 183, 03019 (2018)

[4] R. Bloniarz, J. Majta, Trujillo, E. K. Cerreta and K. Muszka, The mechanisms for strengthening under dynamic loading for low carbon and microalloyed steel, Int. J. Impact Eng., Vol. 114, no. 2017, pp. 53-62 (2018).

[5] A. H. Cottrell and B. A., Bilby Dislocation Theory of Yielding and Strain Ageing of Iron, Proc. Phys. Soc. A., vol. 62, pp. 49 (1949). 